\title{
Sampled Data Systems Passivity and Discrete Port-Hamiltonian Systems
}

\author{
Stefano Stramigioli, Senior Member, IEEE, Cristian Secchi, Member, IEEE, Arjan J. van der Schaft, Fellow, IEEE, \\ and Cesare Fantuzzi, Member, IEEE
}

\begin{abstract}
In this paper, we present a novel way to approach the interconnection of a continuous and a discrete time physical system first presented in [1]-[3]. This is done in a way which preserves passivity of the coupled system independently of the sampling time $T$. This strategy can be used both in the field of telemanipulation, for the implementation of a passive master/slave system on a digital transmission line with varying time delays and possible loss of packets (e.g., the Internet), and in the field of haptics, where the virtual environment should 'feel' like a physical equivalent system.
\end{abstract}

Index Terms-Haptics, Sampled passivity, sampled port-Hamiltonian systems, telemanipulation.

\section{INTRODUCTION}

$\mathbf{T}$ HE USE of network theoretical concepts and passivity is of great importance not only in electrical circuits, but also in robotics [4]-[6]. Recently, a very general and mathematically elegant way to handle general nonlinear networks in a geometrical way has been introduced which is based on Dirac structures [7]-[11]. Systems described in this form are called port-Hamiltonian. The port-Hamiltonian formalism is able to describe any physical system using the mathematical object of a Dirac structure together with some elements which do represent storage, dissipation and interaction of the system with the rest of the world. The interconnection of these parts is based on the concept of a power port which is rich enough to describe spatial mechanism interconnections [11] or even flexible structures [12]. The main idea stems from bond graphs introduced by Paynter [13] which has been incredibly enriched by a proper mathematical description and analysis. The importance of port-Hamiltonian theory has also been recognized by the European Union which recently sponsored a project called Geoplex (http://www.geoplex.cc) whose goal is to study these kind of systems.

Port-Hamiltonian systems have always been described and used in continuous time which is not sufficient for real time control applications like haptics and telemanipulation. The first time that this setting has been extended to sampled data systems can be found in [1] of which this paper is an extension. In this

Manuscript received June 7, 2003; revised October 24, 2003 and May 14, 2004. This work has been done in the context of the European sponsored project GeoPlex with reference code IST-2001-34166. This paper was recommended for publication by Associate Editor K. Lynch and Editor H. Arai upon evaluation of the reviewer's comments.

S. Stramigioli and A. J. van der Schaftis are with the Drebbel Institute of Mechatronics, University of Twente, Enschede, NL-7500, AE, The Netherlands; (e-mail: S.Stramigioli@ieee.org, A.J.Vanderschaft@math.utwente.nl).

C. Secchi and C. Fantuzzi are with the Dipartimento di Scienze e Metodi dell'Ingegneria, University of Modena and Reggio Emilia, 42100 Reggio Emilia, Italy (e-mail: secchi.cristian@unimore.it; fantuzzi.cesare@ unimore.it). Digital Object Identifier 10.1109/TRO.2004.842330 paper it is shown that the usage of a proper definition of sampled data system passivity can lead to simple and powerful results which lead to the passive interconnection of a physical robotic system with a control loop preserving passivity under interaction with an unknown passive environment, a human operator for a haptic interface. These results can be extended easily also to telemanipulation using ideas based on geometric scattering [14], but now, thanks to the material introduced, this is possible for time varying time delays like in Internet applications. Haptics and Tele-manipulation are both characterized by the physical interaction of humans with robotic systems. The importance of passivity, port based models and the description of the interaction using physical elements as springs and dampers have been widely recognized in the literature due to the fact that humans are highly unstructured. Port-Hamiltonian system theory is a reasonable novel theory which allows to analyze physical systems interaction in a much more general way that it would be possible with simple physical models. Especially for robotics, where geometry plays an important role, port-Hamiltonian systems can be very useful as for example also shown in [11]. Furthermore, the authors believe that the basic conceptual idea presented in this paper could be slightly adapted and used for discretization of the PVFC algorithm presented in [15]. For example, the skew symmetric mapping appearing in [15], which has also been considered previously as a passive compensation in [16], could be implemented in the same kind of way with the only difference that, as it could be seen, the modulation due to $q$ in [15] would have to be kept constant between samples. This would not prevent passivity since its skew symmetry would be preserved, but the achieved vector field could be slightly different. Furthermore, the mismatch of energy at posteriori should then be taken into account in the controller energy storage.

The paper is organized as follows. In Section II we will give some background on port-Hamiltonian systems and in Section III we will see how to discretize these systems preserving their energetic properties and how to interconnect, in a passive way, continuous and discrete port-Hamiltonian systems. In Sections IV and V we will illustrate how to use discrete port-Hamiltonian system and discrete-time scattering in the framework of telemanipulation and haptics respectively. In Section VI we will provide some simulations in order to validate our results and we will end with some concluding remarks in Section VII.

\section{BACKGROUND}

In this section we try to give an intuitive description of portHamiltonian systems using coordinates in order to concentrate 
on the prime contribution of the paper. More formal descriptions can be found in [17]. We can consider a port-Hamiltonian system as composed of a state manifold $\mathcal{X}$, an energy function $H: \mathcal{X} \rightarrow \Re ; x \mapsto H(x)$ corresponding to the internal energy, a network structure matrix $D(x)=-D(x)^{T}$ whose graph has the mathematical structure of a Dirac structure [7], which is in general a state dependent power continuous interconnection structure and an interconnection port represented by an effort-flow pair $(e, f) \in V^{*} \times V$ which is geometrically characterized by dual vector elements. This port is used to interact energetically with the system. The power supplied through a port is equal to $e(f)$ or using coordinates to $e^{T} f$. We can furthermore split the interaction port in more sub-ports, each of which can be used to model different power flows. We will indicate with the subscript $I$ the power ports by means of which the system interacts with the rest of the world, with the subscript $C$ the power ports associated with the storage of energy and with the subscript $R$ the power ports relative to the dissipative part.

Summarizing, we have

$$
\left(\begin{array}{c}
e_{I} \\
f_{C} \\
e_{R}
\end{array}\right)=D(x)\left(\begin{array}{c}
f_{I} \\
e_{C} \\
f_{R}
\end{array}\right)
$$

where

$$
D(x):=\left(\begin{array}{ccc}
D_{I} & G_{1} & G_{2} \\
-G_{1}^{T} & D_{C} & G_{3} \\
-G_{2}^{T} & -G_{3}^{T} & D_{R}
\end{array}\right)
$$

and $D_{I}, D_{C}, D_{R}$ are skew-symmetric. Due to the skew-symmetry of $D(x)$, we clearly have, using coordinates

$$
P_{I}+P_{C}+P_{R}:=e_{I}^{T} f_{I}+e_{C}^{T} f_{C}+e_{R}^{T} f_{R}=0
$$

which is a power balance representing Tellegen's theorem and meaning that the total power coming out of the network structure should be always equal to zero. A dissipating element of the system can be modeled using as characteristic equations $e_{R}=R(x) f_{R}$ with $R(x)$ a symmetric semidefinite matrix. This implies that

$$
f_{R}=\left(D_{R}-R\right)^{-1} G_{2}^{T} f_{I}+\left(D_{R}-R\right)^{-1} G_{3}^{T} e_{C}
$$

and therefore

$$
\left(\begin{array}{c}
e_{I} \\
f_{C}
\end{array}\right)=\left(\begin{array}{ll}
B & A \\
C & D
\end{array}\right)\left(\begin{array}{c}
f_{I} \\
e_{C}
\end{array}\right)
$$

where

$$
\begin{aligned}
B & :=D_{I}+G_{2}\left(D_{R}-R\right)^{-1} G_{2}^{T} \\
A & :=G_{1}+G_{2}\left(D_{R}-R\right)^{-1} G_{3}^{T} \\
C & :=-G_{1}^{T}+G_{3}\left(D_{R}-R\right)^{-1} G_{2}^{T} \\
D & :=D_{C}+G_{3}\left(D_{R}-R\right)^{-1} G_{3}^{T}
\end{aligned}
$$

If we furthermore set $\dot{x}=f_{C}$ and $e_{C}=(\partial H) /(\partial x)$, due to the previous power balance we obtain

$$
\dot{H}+f_{R}^{T} R(x) f_{R}=-e_{I}^{T} f_{I}
$$

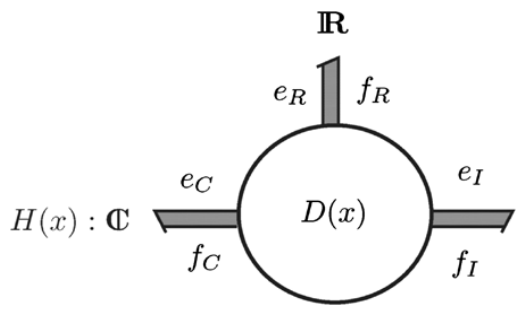

Fig. 1. Interconnection description.

which clearly says that the supplied power $-e_{I}^{T} f_{I}$ equals the increase of internal energy plus the dissipated one.

A schematic diagram of the system is reported in Fig. 1 where the half arrows direction indicate the positive oriented direction of power, $\mathbf{C}$ indicates a storage of energy, and $\mathbf{R}$ indicates the dissipation of free-energy using bond graphs notation [11]. Any lumped physical system can be modeled using this structure or extensions of it. Even for distributed parameter systems these ideas can be extended [18].

Throughout the paper we will use the following definition of passivity.

Definition 1: A system is passive if the supplied power is either stored (with a lower bounded storage function) or dissipated.

It follows directly from the definition that a system is passive if

$$
P=e^{T} f=\frac{d E}{d t}+P_{\text {diss }}
$$

where $P$ represents the power supplied through a power port, $E$ a lower bounded function representing the stored energy, and $P_{\text {diss }} \geq 0$ the dissipated power. If $P_{\text {diss }}<0$, it means that there is production of some extra energy and that, therefore, the system is not passive. A port-Hamiltonian system represented by (2) is passive. In fact, the supplied power is $P=-e_{I}^{T} f_{I}$ and passivity directly follows from (7).

As a simple comprehensive one dimensional example, consider a parallel connected linear spring with stiffness $k$, and a damper with damping coefficient $b$. We can interact with the system by pushing the two interconnected elements. The energy of the system is $H(x)=(1 / 2) k x^{2}$ which represents the potential energy of the spring having as state the displacement $x ; f_{C}$ is then by definition $\dot{x}$ and $e_{C}=k x$ which is equal to the elastic force. As expected, the change in potential energy is $e_{C} f_{C}$. The damping effect can be modeled with $e_{R}=b f_{R}$. The applied motion would be $f_{I}=v$, the felt reactive force $e_{I}=-F$ and the supplied power by the external force $e_{I} f_{I}$. For this system, the total equations become

$$
\left(\begin{array}{c}
-F \\
\dot{x} \\
b f_{R}
\end{array}\right)=\left(\begin{array}{ccc}
0 & -1 & -1 \\
1 & 0 & 0 \\
1 & 0 & 0
\end{array}\right)\left(\begin{array}{c}
v \\
k x \\
f_{R}
\end{array}\right) .
$$

Clearly the matrix $D(x)$ is skew-symmetric as expected. The total power flowing in the system is given by

$$
\begin{aligned}
P_{I}+P_{C}+P_{R} & =-F v+\dot{x} k x+f_{R} b f_{R} \\
& =\left(\begin{array}{lll}
-F & \dot{x} & b f_{R}
\end{array}\right)\left(\begin{array}{c}
v \\
k x \\
f_{R}
\end{array}\right) .
\end{aligned}
$$


Using (8) we obtain that

$$
P_{I}+P_{C}+P_{R}=\left(\begin{array}{lll}
v & k x & f_{R}
\end{array}\right)\left(\begin{array}{ccc}
0 & 1 & 1 \\
-1 & 0 & 0 \\
-1 & 0 & 0
\end{array}\right)\left(\begin{array}{c}
v \\
k x \\
f_{R}
\end{array}\right)=0
$$

where the last equality follows from the skew symmetry of the matrix $D(x)$ and shows that Tellegen's theorem, represented by (1), is satisfied. With the steps reported previously it is possible to get the form reported in (2)

$$
\left(\begin{array}{c}
-F \\
\dot{x}
\end{array}\right)=\left(\begin{array}{cc}
-b & -1 \\
1 & 0
\end{array}\right)\left(\begin{array}{c}
v \\
k x
\end{array}\right) \text {. }
$$

\section{SAMPLED DATA Systems PASSIVITY}

\section{A. Discrete Port-Hamiltonian Systems}

We can describe a discrete time port-Hamiltonian system as a continuous time port-Hamiltonian system in which the port variables are frozen for a sample interval $T$. In what follows we indicate with $v(k)$ the value of the discrete variable $v(t)$ corresponding to the interval $t \in[k T,(k+1) T]$. If we rewrite (1) for the discrete case, we have

$$
e_{I}^{T}(k) f_{I}(k)+e_{C}^{T}(k) f_{C}(k)+e_{R}^{T}(k) f_{R}(k)=0 .
$$

Furthermore, during the interval $k$, we have to consider a constant state $x(k)$ corresponding to the continuous time state $x(t)$. This implies that during the interval $k$, the dissipated energy will be equal to $T f_{R}^{T}(k) R(x(k)) f_{R}(k)$ and the supplied energy will be equal to $-T e_{I}^{T}(k) f_{I}(k)$. In order to be consistent with the energy flows, and as a consequence conserve passivity, we need therefore a jump in internal energy $\Delta H(k)$ from instant $k T$ to instant $(k+1) T$ such that

$$
\Delta H(k)=-T f_{R}^{T}(k) R(x(k)) f_{R}(k)-T e_{I}^{T}(k) f_{I}(k) .
$$

This implies that the new discrete state $x(k+1)$ should belong to an energetic level such that

$$
H(x(k+1))=H(x(k))+\Delta H(k) .
$$

We can indicate the set of possible energetically consistent states as

$$
I_{k+1}:=\{x \in \mathcal{X} \text { s.t. } H(x)=H(x(k))+\Delta H(k)\} .
$$

Furthermore, from the discrete equivalent of (2), we have that

$$
f_{C}(k)=C f_{I}(k)+D e_{C}(k)
$$

and therefore, for consistency with the continuous dynamics in which $f_{C}(t)=\dot{x}(t)$, the next state $x(k+1)$ should be such that

$$
f_{C}(k)=\lim _{T \rightarrow 0} \frac{x(k+1)-x(k)}{T}
$$

where we considered the definition of the right derivative.

Remark 1: It is important to notice that the choice of the next discrete state which is taken right after sampling instant $k+1$, can be done causally based on the energy exchange which has taken place in the last interval between sampling $k$ and $k+1$.

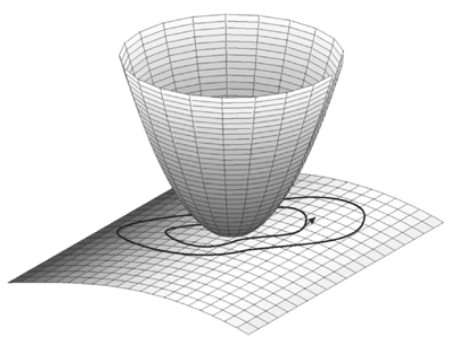

Fig. 2. The passivity-preserving energetic behavior of a discrete port-Hamiltonian system.

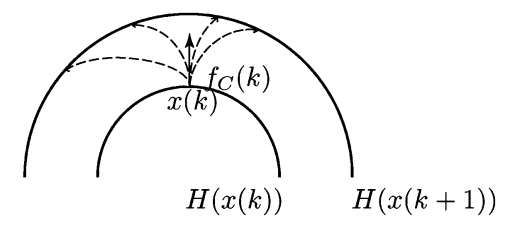

Fig. 3. Various possible connections for the state jump.

The set $I_{k+1}$ can be either empty or have more solutions.

1) Case $I_{k+1} \neq \emptyset$ : This situation is the most common and corresponds to the normal one. A graphical representation is shown in Fig. 2 where two energy levels corresponding to the plotted energy function are given. In this case a choice should be made among the possible states of $I_{k+1}$. Clearly, the state should be in some sense 'close' to the current state $x(k)$ and such that the condition of (13) is satisfied. A picture which shows graphically the basic idea is reported in Fig. 3. The possible curves going through $x(k)$ and having as a tangent $f_{C}(k) \in T_{x(k)} \mathcal{X}$, could be characterized as geodesics once an affine connection would be defined on $\mathcal{X}$. These possible different geodesics are indicated as dotted lines in the figure. These lines intersect the locus of states corresponding to the consistent energy set in different possible points which would depend on the connection chosen. In this paper we have considered Euclidean coordinates and the Euclidean connection associated to them. In this case, the next state $x(k+1)$ is chosen as the intersection of $I_{k+1}$ with the straight line passing from $x(k)$ and directed along $f_{C}(k)$.

2) Case $I_{k+1}=\emptyset$ and Energy Leap: This can happen in two situations a) required decrease of energy close to a local minimum or b) required increase of energy close to a local maximum. The case b) does not preclude passivity and therefore will not be analyzed in this paper.

In the situation a) let us indicate with $x_{\min }$ one of the states for which the energy has locally a minimum close to $x(k)$ and equal to $H\left(x_{\min }\right)$. This situation is therefore obtained if

$$
\Delta H(k)<H\left(x_{\min }\right)-H(x(k)) .
$$

In this situation, it is clearly not possible to find a state $x(k+$ 1) compatible with the energy change $\Delta H(k)$ since $I_{k+1}=$ $\emptyset$. If we chose $x(k+1)=x_{\min }$ we would implement the smallest error in the energy change, but this would not be a good choice for two main reasons: First, this could create a "dynamic 


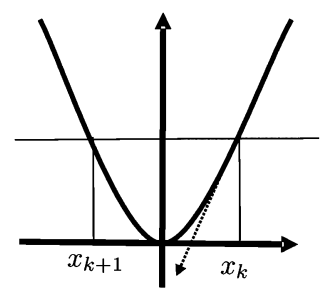

Fig. 4. Choice of the next state in the case in which $I_{k+1}=\emptyset$.

dead-lock" since, in this situation the effort generated by the energy function and equal to $e_{C}=(\partial H) /(\partial x)$ would be equal to zero and in case no damping would be present, it would be possible to see that this would prevent any further supply of energy from the interconnection port $\left(e_{I}, f_{I}\right)$ since $e_{I}$ could be equal to zero and therefore any further change of the state would be impossible. Second, it would not help the system to behave in such a way that its dynamic would make possible to correct the energetic discrepancy created since the required $\Delta H(k)$ cannot be performed. A solution to these two problems can be found in what we call energy leap which is illustrated in Fig. 4. Instead of choosing as a new state $x_{\min }$, we choose as a state $x(k+1)$, a state with the same energy level, but 'symmetrically positioned' with respect to the point of minimum energy $x_{\min }$. This rather fuzzy statement could be made precise once an affine connection would be defined on the state manifold. As already said, considering Euclidean coordinates, it is possible to define the next state as the state having the same energy and lying on a straight line passing through $x_{\min }$ and $x(k)$.

Clearly, by construction, we obtain an error in the energy change which can be as big as $\Delta H(k)$ which corresponds to the amount of energy which we supplied to the "rest of the world" through the power port $\left(e_{I}, f_{I}\right)$. On the other hand, by the change of sign in the gradient of the energy function, we practically passed through the minimum in one go and the system will therefore now try to absorb energy from the port $\left(e_{I}, f_{I}\right)$. This will then often yield to an increase of the internal energy. It would therefore then be possible to increase the internal energy not exactly with the required amount but with inferior values. In such a way, it would be possible using energy error book-keeping to maintain passivity of the discrete system using an horizon longer than a sample period.

Formally, we can consider a discrete variable $\bar{H}(k)$ representing the energy which has been supplied to the rest of the universe which was not available internally. If $I_{k+1}=\emptyset$, we will set $\bar{H}(k+1)=\bar{H}(k)+\Delta H(k)$. On the other hand, if $I_{k+1} \neq \emptyset$, we can modify the net increase or decrease of the real stored internal energy $H(k)$ by "diverting" some energy in order to decrease $\bar{H}(k)$. If the calculated $\Delta H(k)$ is positive, it means that energy has been flowing in from the environment. In that case, we can calculate the next discrete state not based on $\Delta H(k)$, but based on $\alpha \Delta H(k)$ with $\alpha \in] 0 \ldots 1$ ] and close to 1 , and at the same time we can decrease $\bar{H}(k+1)=\bar{H}(k)-(1-\alpha) \Delta H(k)$. The closer is $\alpha$ to 1 the slower the "passivity recovery" would be $(\bar{H}(k) \rightarrow 0)$ and the less influence will have the dynamics. In case $\Delta H(k)$ is negative, it is possible to decrease $\bar{H}(k)$ by temporarily enabling some dissipation on the interconnection port of the sampled system. The major point is that it is possible to

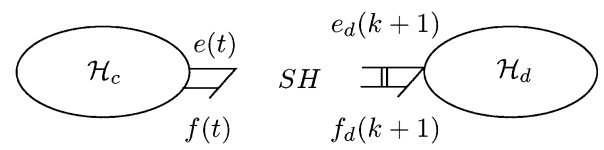

Fig. 5. Interconnection of discrete and continuous port-Hamiltonian systems.

keep track exactly of the error in the energy values in such a way that they can be then compensated.

As a summary of the procedure just outlined, we hereafter algorithmically explain the way the discrete system can be integrated

1) Given an initial state $x(k)$, we set $e_{C}(k)=$ $(\partial H) /(\partial x)\left(x_{k}\right)$.

2) Using the value of the system input $f_{I}(k)$ and the previously calculated $e_{C}(k)$, we can calculate $e_{I}(k)$, the output of the interaction port, and $f_{C}(k)$ using the discrete representation of (2) computed at the sampling instant $k T$.

3) $f_{C}(k)$ is then used to calculate the next state $x(k+1)$ using the procedure explained at the beginning of this subsection.

\section{B. Energy Consistent Sampled Passivity}

In this section, we will expose a scheme, first introduced in [1], to passively interconnect continuous and discrete time interacting systems. Consider the port interconnection of a continuous time Hamiltonian system $H_{C}$ and a discrete Hamiltonian system $H_{D}$ (the result of this subsection is independent of the nature of the energetically interconnected systems) through a sampler and zero-order hold as shown in Fig. 5 in a bond-graph notation. The double band on the bond indicates a "virtual" energetic exchange in the discrete domain. The pair $(e(t), f(t))$ forms the interconnection port of the continuous system $H_{C}$; we omit the subscrit $I$ to indicate the interconnection port to avoid confusion with the discrete interconnection port of the system $H_{D}$ which will be represented by the pair $\left(e_{I}(k), f_{I}(k)\right)$. Suppose that $H_{C}$ has an admittance causality (effort in/flow out) and therefore $H_{D}$ has an impedance causality (flow in/effort out). During the dynamic evolution of the two systems between time $k T$ and $(k+1) T$, where $T$ is the sampling time and $k$ is a positive integer, the effort supplied to $\mathcal{H}_{C}$ by $\mathcal{H}_{D}$ will be constant due to the zero-order hold assumption. We will indicate this value as $e_{d}(k+1)$

$$
e(t)=e_{d}(k+1) \quad t \in[k T(k+1) T] .
$$

By looking at the energy flow toward the continuous system, we can see that, if we indicate with $\Delta H_{C}^{\text {in }}(k+1)$ the energy which flows through the input power port from time $k T$ up to time $(k+1) T$, we obtain

$$
\begin{aligned}
\Delta H_{C}^{\mathrm{in}}(k+1) & =\int_{k T}^{(k+1) T} e_{d}^{T}(k+1) f(s) d s \\
& =e_{d}^{T}(k+1) \int_{k T}^{(k+1) T} f(s) d s \\
& =e_{d}^{T}(k+1)(q((k+1) T)-q(k T))
\end{aligned}
$$

where we indicated with $q(\cdot)$ the integral of the continuous time flow $f(t)$. 
Remark 2: It is important to realize that, in most of useful mechanical applications like haptics or telemanipulation, $e_{d}(k+$ 1) will correspond to forces/moments that a controller would apply to an inertial element. In this case, $q(\cdot)$ would be nothing else than a position measurement of the masses the controller pushes on.

It is now straightforward to state the following theorem.

Theorem 1 (Sample Data Passivity): If in the situation sketched before, we define for the interconnection port of $H_{D}$

$$
f_{d}(k+1):=\frac{q(k T)-q((k+1) T)}{T},
$$

we obtain an equivalence between the continuous time and discrete time energy flow in the sense that for each $n$

$$
\sum_{i=0}^{n-1} e_{d}^{T}(i+1) f_{d}(i+1)=-\int_{0}^{n T} e^{T}(s) f(s) d s
$$

Remark 3: It is important to notice that the exact equivalence is achieved only by the definition of (15) in which $q(\cdot)$ is usually the easiest variable to be measured in real applications. The negative sign appearing in (16) is consistent with the fact that the power flowing into the continuous system is minus the power flowing into the discrete side. It may happen that during the inter-sample, passivity of the controller is lost without that the controller would find this out. This could be due to a sequence of a very high flow of energy toward the plant followed by the same amount of energy back to the controller. This would be a problem because the net energy flow toward the plant after one sample would be zero, but between the first and the second peak, there would be a moment in which the controller would have provided more energy that it had available. This "loss of passivity" between the two spikes would never be seen by the controller since this situation takes place between two samples. This situation is unavoidable because of the intrinsic loss of information due to sampling if no hypothesis is made on continuity or bandwidth, but at each sample instant the controller would adjust the energy balance as explained in the previous section.

It is now possible to propose the algorithm which can be used to integrate the discrete system on line. We consider to reach time $(k+1) T$ after a sample period in which the effort held on the device has been $e_{d}(k+1)$. After $q(k+1)$ has been measured, $f_{d}(k+1)$ can be calculated using (15). If we consider the situation at the previous time $k$ and define $f_{I}(k):=f_{d}(k+1)$, we are able to calculate the state $x(k+1)$ by exactly following the algorithm of the previous section and the value $e_{I}(k)$ that the system should have provided, but that couldn't provide because future information, only available at time $(k+1) T$, was necessary to compute $e_{I}(k)$ using (2), through the hold instead of $e_{d}(k+1)$ in the last interval. It is important to realize that the calculated state $x(k+1)$ is exactly compatible with the energy balance by construction using the result of (16). The only problem is the fact that the held value $e_{d}(k+1)$ is not equal to the value $e_{I}(k)$ which would have been expected by the use of discrete representation of (2) at the sample instant $k T$. On the other hand, we can set $e_{d}(k+2)=e_{I}(k)$ and use the correct value with a delay $T$. This operation does NOT break the passivity of the algorithm and it is possible to see that it will have little influence on the dynamics for reasonably small $T$ since the acceleration profile will be roughly shifted by $T$ and the inertial properties of the robotic device will behave like a low pass filter. It is then possible to calculate $e_{C}(k+1)$ using the value of $x(k+1)$ and then, when time $(k+2) T$ is reached, to start over the algorithm for computing the next state.

1) Deadlock Effect and Its Avoidance: The presented strategy works fine in all situations in which the state $x(k)$ is not on a minimum of the storage function $H($.$) . Problems arise$ instead, if at any moment of time the state $x(k)$ is indeed in a minimum. To see this, consider the simulation of a purely one-dimensional, linear, elastic element with internal energy $H(x)=(1 / 2) K x^{2}$. Suppose a starting unloaded rest situation $(x(0)=0)$ and $e_{d}(1)=0$. Due to the fact that $x(0)$ is in a minimum, $e_{C}(0)=0$. Consider that a user would apply a motion to the robotic device in such a way that at time $1, f_{d}(1)$ would result different than zero. Due to the fact that the supplied effort $e_{d}(1)=0$, the exchanged power between the robotic device and the Hamiltonian controller is equal to zero and this would result again in $x(1)=0$. The problem arises due to the fact that in this example, $e_{I}(0)=e_{C}(0)=0$ and this implies that $e_{d}(2)$ will again be zero. This would result in a constant zero force applied to the robotics device which is clearly not what we wanted. This seemingly critical shortcoming of the algorithm can be nevertheless easily corrected, by modifying the choice of $e_{d}$ for the next step

$$
e_{d}(k+2)=\frac{e_{I}(k)+\bar{e}_{I}(k)}{2}
$$

where $\bar{e}_{I}(k)$ can be taken as the value of the port effort calculated with a first order Euler approximation of the continuous time system which we want to haptically display. In the case of the spring, this would be

$$
\bar{e}_{d}(k+1)=K f_{d}(k+1) .
$$

This addition can be easily generalized for the extended structure of a general physical system.

\section{Passive Coupled Behavior}

From the previous considerations, it is possible to understand that at each sampling time, we have an exact matching between the physical energy going to the continuous time system and the virtual energy coming from the discrete time port independently of the sample time $T$ and of eventual intersample dynamics of the continuous system. It is remarkable that the choice reported in (15) which is very simple and at the same time attractive due to the fact that it corresponds to position measurements, in practice gives such a powerful and at the same time trivial result.

This means that we can passively interconnect the two systems in such a way that independently of the sampling time and its relation with the characteristics of the interconnected systems, the two systems would be energetically consistent at each sampling time and no energy would be created by the sampling and hold procedure. 


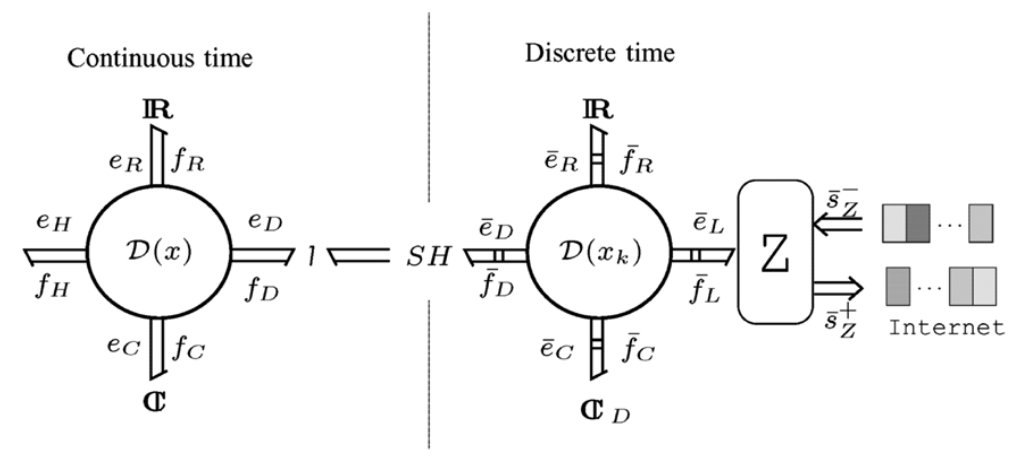

Fig. 6. Passive Sample Data Telemanipulation Scheme.

The implementation of a passivity preserving interconnection is independent of the nature of the systems interconnected. Nevertheless the behavior of the overall system depends on the structure of the interconnected systems. Since continuous and discretized (using the algorithm proposed in Section III-A) portHamiltonian systems are passive, their interconnection leads to an overall system that is characterized by a passive behavior.

The only energy leakage is due to the fact that the discrete time system has no way whatsoever to predict the value of the continuous time system at the interconnection port and this implies that only at the end of the sample period will have an exact measure of the energy it supplied to the continuous time system.

This is due to the fact that with a fixed force set-point kept constant for the all sampling interval and no assumption on the dynamics of the haptic device and human, any velocity profile is in principle allowed and therefore also any energy exchange between the controller and the haptic device during the sampling interval since the controller will not react until next sample.

This gives rise to the problems reported in Section III-A2. These are structural problems due to the fact that the data obtained by sampling can never give enough information in order to causally reconstruct the physical behavior of the interconnection as it would happen with an acausal Shannonreconstructor.

They can be either compensated by a clever book-keeping of the energy in excess supplied to the continuous time system or by a continuous time damping circuit which can be easily built with a couple of operational amplifiers directly connected to the power amplifiers used to drive the continuous time system. In the PO/PC scheme proposed by Hannaford [4] a similar approach is used for coupling discrete and continuous time interacting systems but no exact measure for the produced energy is used and this leads to some assumptions on the sampling rate.

\section{TElemanipulation}

In the design of a control system for telemanipulation there are two main problems to be addressed in order to avoid instability and poor performance: the communication channel and the interaction with an unknown environment.

The first problem has been approached in several manners for fixed time delays in continuous time using scattering theory [5], [6]. Scattering theory has been also used in [19], [20] to build bilateral telemanipulation systems, in [21] for dealing with the variable delay problem and in [22] to build a passive position controlled teleoperator. A very suitable tool to solve the second problem is passivity theory and the control of the energy exchange between the interacting systems using IPCs [11]. In [14] a general, geometric coordinate-free way framework for the telemanipulation of port-Hamiltonian systems in the continuous time case is proposed which has been extended in [23] for grasping applications. For an overview and a comparison of the various techniques proposed in the literature, see, for instance, [24] and [25].

The schemes consider continuous time controllers and fixed delay communication channels. The sampled data nature of the controllers can be considered by using the concepts described in Section III. In fact, we can use the discretization algorithm reported in Section III-A in order to obtain a discrete passive IPC and to interconnect it by means of the energetic consistent interconnection described in Section III-B to the continuous plant. Since now the controllers connected to the transmission line are discrete we have to define a digital transmission line through which master and slave side will exchange energy. The proposed scheme is illustrated in Fig. 6 in a bond-graph notation [11]. The subscript $H$ indicates the power port by means of which the system interacts with the rest of the world, the subscript $R$ represents the dissipative port while the subscript $C$ the port associated to a storage of energy. The subscript $L$ indicates the power port associated to the communication channel and the subscript $D$ the power port of interaction between the system and the controller. The barred power variables and the barred bonds represent discrete power variables and discrete energy exchange respectively. Both continuous and discrete systems are, generally, nonlinear systems.

\section{A. Discrete-Time Scattering and Energy Packets}

The aim of this section is to define a discrete-time scattering based communication channel and to analyze its energetic behavior; this problem has been first addressed in [2], [3]. Consider the discrete-time port $\left(\bar{e}_{L}, \bar{f}_{L}\right)$ of Fig. 6 which is connected to the transmission line. The energy flowing into the system in the interval $[k T,(k+1) T]$ is equal to

$$
E_{\text {line }}=T \bar{e}_{L}^{T}(k) \bar{f}_{L}(k) .
$$

Furthermore, if we consider the geometric scattering formalism for continuous time introduced in [14], we have that using the scattering decomposition, at each instant of time, we have the 


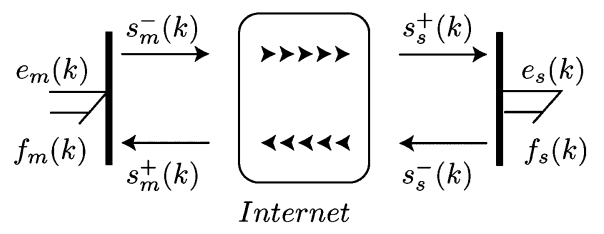

Fig. 7. Scatterized communication channel.

following important power balance which is at the base of the scattering formalism

$$
e^{T} f=\frac{1}{2}\left\|s_{Z}^{+}\right\|^{2}-\frac{1}{2}\left\|s_{Z}^{-}\right\|^{2}
$$

where

$$
\left\{\begin{array}{l}
s_{Z}^{+}=\frac{N^{-1}}{\sqrt{2}}(e+Z f) \\
s_{Z}=\frac{N^{-1}}{\sqrt{2}}(e-Z f)
\end{array}\right.
$$

and $Z=N N$ is a positive definite matrix representing the impedance of the scattering transformation. We can interpret $s_{Z}^{+}$

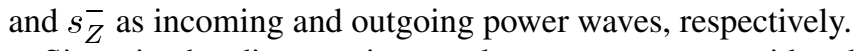

Since in the discrete time analogous we can consider the system as having the port variables frozen between one sample time and the next, we can multiply the previous equation by the sample time $T$ and obtain

$$
E_{\text {line }}(k)=\frac{T}{2}\left\|s_{Z}^{+}(k)\right\|^{2}-\frac{T}{2}\left\|s_{Z}^{-}(k)\right\|^{2}
$$

where $T$ is the sample period. Thus, we can interpret $(T / 2)\left\|s_{Z}^{+}\right\|^{2}$ and $(T / 2)\left\|s_{Z}^{-}\right\|^{2}$ as incoming and an outgoing energy packets respectively.

At each sample time the system will acquire the incoming energy quantum $(T / 2) s_{Z}^{+}(k)$ and the discrete effort $e(k)$ and will calculate the discrete flow $f(k)$ and the discrete energy quantum $(T / 2) s_{Z}^{-}(k)$ to transmit through the communication channel. In [14] the mappings which allow to compute $s_{Z}(k)$ and $f(k)$ from $s_{Z}^{+}(k)$ and $e(k)$ are reported.

1) Constant Time Delay: Let us now analyze the energetic behavior of the digital scattering-based communication channel. We will drop the subscript $Z$ from the scattering variables (assuming that a proper line impedance has been fixed) and we will refer to the discrete communication channel represented in Fig. 7. We will use the following notation for the discrete derivative and the discrete integral:

$$
d g(k)=\frac{g(k+1)-g(k)}{T} \quad I_{h}^{k} g=\sum_{i=h}^{k-1} g(i) T
$$

where $g$ is a generic sequence.

Proposition 1: In case of fixed transmission delays and no loss of packets, the discrete communication channel is lossless in a discrete sense.

Proof: The power flow into the communication channel is

$$
\begin{aligned}
P_{L}(k)=\frac{1}{2}\left\|s_{m}^{-}(k)\right\|^{2}+ & \frac{1}{2}\left\|s_{s}^{-}(k)\right\|^{2} \\
& -\frac{1}{2}\left\|s_{m}^{+}(k)\right\|^{2}-\frac{1}{2}\left\|s_{s}^{+}(k)\right\|^{2}
\end{aligned}
$$

but

$$
s_{s}^{+}(k)=s_{m}^{-}\left(k-d_{m}\right) \quad s_{m}^{+}(k)=s_{s}^{-}\left(k-d_{s}\right)
$$

where $d_{m}$ and $d_{s}$ are respectively the delays associated to the communication between master and slave and slave and master respectively. We can therefore write.

$$
\begin{aligned}
P_{L}(k)=\frac{1}{2}\left\|s_{m}^{-}(k)\right\|^{2}-\frac{1}{2}\left\|s_{m}^{-}\left(k-d_{m}\right)\right\|^{2} & \\
& \quad+\frac{1}{2}\left\|s_{s}^{-}(k)\right\|^{2}-\frac{1}{2}\left\|s_{s}^{-}\left(k-d_{s}\right)\right\|^{2}
\end{aligned}
$$

By trivial computations it can be shown that, in general

$$
d I_{k-h}^{k} g=g(k)-g(k-h)
$$

where $g$ is a generic sequence. We can, therefore, write

$$
P_{L}(k)=d\left[I_{k-d_{m}}^{k}\left(\frac{1}{2}\left\|\left(s_{m}^{-}\right)\right\|^{2}\right)+I_{k-d_{s}}^{k}\left(\frac{1}{2}\left\|\left(s_{s}^{-}\right)\right\|^{2}\right)\right]
$$

The power $P_{L}(k)$ of the communication channel represents the variation of the discrete energy stored into the communication channel. Since $P_{L}(k)$ can be written as the discrete time derivative, as shown in the previous equation, it is possible to define the discrete energy stored into the communication channel as

$$
E_{L}(k)=\left[I_{k-d_{m}}^{k}\left(\frac{1}{2}\left\|s_{m}^{-}\right\|^{2}\right)+I_{k-d_{s}}^{k}\left(\frac{1}{2}\left\|s_{s}^{-}\right\|^{2}\right)\right]
$$

we have that

$$
P_{L}(k)=d E_{L}(k)
$$

which means that all the power flowing through the communication channel is stored. The communication channel is, therefore, lossless in a discrete sense.

In practical applications, packet switching communication channel (e.g., Internet) are unreliable because it can happen that some packets could be lost during transmission because of some traffic problems or some troubles in the servers each packet has to cross. Let us investigate the energetic behavior of the communication channel in the case one packet is lost in the transmission between master and slave. We will have that a 0 packet is received at the slave side instead of the correct value, namely $s_{m}^{-}\left(k-d_{m}\right)$ and that

$$
P_{L}(k)=\frac{1}{2}\left\|s_{m}^{-}(k)\right\|^{2}+\frac{1}{2}\left\|s_{s}^{-}(k)\right\|^{2}-\frac{1}{2}\left\|s_{m}^{+}(k)\right\|^{2} .
$$

We can write

$$
\begin{aligned}
P_{L}(k)= & \frac{1}{2}\left\|s_{m}^{-}(k)\right\|^{2}+\frac{1}{2}\left\|s_{s}^{-}(k)\right\|^{2}-\frac{1}{2}\left\|s_{m}^{+}(k)\right\|^{2} \\
= & \frac{1}{2}\left\|s_{m}^{-}(k)\right\|^{2}+\frac{1}{2}\left\|s_{s}^{-}(k)\right\|^{2}-\frac{1}{2}\left\|s_{s}^{-}\left(k-d_{s}\right)\right\|^{2} \\
& -\frac{1}{2}\left\|s_{m}^{-}\left(k-d_{m}\right)\right\|^{2}+\frac{1}{2}\left\|s_{m}^{-}\left(k-d_{m}\right)\right\|^{2} \\
= & d E_{L}(k)+\frac{1}{2}\left\|s_{m}^{-}\left(k-d_{m}\right)\right\|^{2}
\end{aligned}
$$

We have an additional term which is positive definite and which represents a dissipated power. When a packet is lost in the transmission the channel dissipates an energy quantum corresponding to the value that it should have received from the master side. The behavior of the system, nonetheless, keeps on being passive. Let, now, $\mathcal{F}_{m s}$ and $\mathcal{F}_{s m}$ the set of time instants in which a packet in the communication between master and slave is lost and the set of time instants in which a packet in the 
communication between slave and master is lost respectively. The energetic behavior of the channel is

$$
\begin{aligned}
P_{L}(k)=d E_{L}(k)+\alpha\left(\frac{1}{2} \| s_{m}^{-}\left(k-d_{m}\right)\right. & \left.\|^{2}\right) \\
+\beta( & \left(\frac{1}{2}\left\|s_{s}^{-}\left(k-d_{s}\right)\right\|^{2}\right)
\end{aligned}
$$

where

$$
\alpha=\left\{\begin{array}{ll}
0, & k \notin \mathcal{F}_{m s} \\
1, & k \in \mathcal{F}_{m s}
\end{array} \quad \beta= \begin{cases}0, & k \notin \mathcal{F}_{s m} \\
1, & k \in \mathcal{F}_{s m}\end{cases}\right.
$$

where $\alpha$ and $\beta$ are coefficients that activate a power dissipation of the channel when a packet gets lost.

2) Variable Time Delay: Let us now consider the case in which the delay between master and slave is variable. Suppose that $h$ is the minimum delay of the transmission between master and slave. Because of the variable delay, it can happen that some packets are so much delayed that the receiving queue is empty for some sample periods. In these cases we can always write

$$
\begin{aligned}
P_{L}(k)= & \frac{1}{2}\left\|s_{m}^{-}(k)\right\|^{2}+\frac{1}{2}\left\|s_{s}^{-}(k)\right\|^{2}-\frac{1}{2}\left\|s_{m}^{+}(k)\right\|^{2} \\
= & \frac{1}{2}\left\|s_{m}^{-}(k)\right\|^{2}+\frac{1}{2}\left\|s_{s}^{-}(k)\right\|^{2}-\frac{1}{2}\left\|s_{s}^{-}\left(k-d_{s}\right)\right\|^{2} \\
& -\frac{1}{2}\left\|s_{m}^{-}(k-h)\right\|^{2}+\frac{1}{2}\left\|s_{m}^{-}(k-h)\right\|^{2} \\
= & d E_{L}(k)+\frac{1}{2}\left\|s_{m}^{-}(k-h)\right\|^{2}=d E_{L}(k)+P_{d}(k)
\end{aligned}
$$

where now we define

$$
E_{L}(k)=\left[I_{k-h}^{k}\left(\frac{1}{2}\left(s_{m}^{-}\right)^{2}\right)+I_{k-d_{s}}^{k}\left(\frac{1}{2}\left(s_{s}^{-}\right)^{2}\right)\right]
$$

as the energy function. We dissipate the energy quantum corresponding to the packet we were expecting. At a time $k+$ $j$ the system receives the packet whose corresponding energy quantum was dissipated at time $k$. In this case it will be

$$
\begin{aligned}
P_{L}(k+j)= & d E_{L}(k+j)-\frac{1}{2}\left\|s_{m}^{-}(k-h)\right\|^{2} \\
& +\frac{1}{2}\left\|s_{m}^{-}(k+j-h)\right\|^{2} \\
= & d E_{L}(k+j)+P_{p}(k+j)+P_{d}(k+j)
\end{aligned}
$$

At sampling period $k+j$, the delayed incoming packet injects an extra energy $T P_{p}(k+j)$, on the other hand the missed packet $(1 / 2)\left\|s_{m}^{-}(k+j-h)\right\|^{2}$, since we are assuming that only one packet can be transmitted per each sample period, causes the dissipation of $T P_{d}(k+j)$ energy.

We can see that the delay of a packet first implies a dissipation of an energy quantum and then an energy injection of the same energy quantum. It is, then, clear that

$$
\sum_{n=0}^{\infty} P_{p}(n)+P_{d}(n)=0
$$

and, therefore, there is no global production of extra energy.

Both in case of fixed and variable delay the scatterized communication channel is lossless; the main difference is that when we have a constant delay energy is neither produced nor dissipated but simply stored, while when we have a variable delay

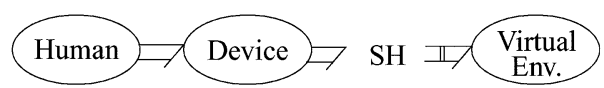

Fig. 8. Energetic representation of a haptic display.

the energy quanta associated to the delayed packets are first dissipated and then injected back to the system. Since the variable delay introduces a finite extra-delay on the packets, passivity is preserved. One could expect that the energy injection leads to some nonpassive (and therefore potentially unstable) behavior but this is not the case since before being injected the extra energy has already been dissipated. In case some packets are lost the behavior of the communication channel is dissipative since we do not have any energy injection to recover the dissipated quanta. It is now straightforward to state the following.

Proposition 2: The scatterized communication channel is passive even in case of variable time delay and of loss of packets.

We can conclude, therefore, that the scheme for telemanipulation proposed in Fig. 6 is intrinsically sampled data passive disregarding loss of packets and variable delays in the communication between master and slave.

\section{HAPTICS}

In the implementation of every control algorithm for interaction tasks, stability is the key issue. Several researches have shown that passivity theory is a very suitable tool for the study of interaction tasks [11]. Furthermore, Hogan [26] proved that the human operator behaves as a passive system in the frequency range of interest in haptics. Colgate [27] has introduced the idea of virtual coupling, to guarantee passivity for arbitrary passive virtual environment and even for a class of nonpassive ones [28]. Hannaford noted that a fixed parameters virtual layer can decrease performances of the system because of an excess of energy dissipation in some working conditions. He introduced a variable virtual layer, loosely speaking a virtual damper which is made active only when some energy production is detected (PO/PC strategy, [4]). Furthermore a lot of studies have been also done on the implementation of virtual environments, see for example [29].

An haptic interface can be modeled as the energetic interconnection of four systems as shown in Fig. 8 where bond-graph notation has been used.

The human operator is a passive system in the range of frequencies of interest. The majority of haptic devices is constituted by mechanical passive systems but their passivity can be gravely affected when the system is actuated. Nevertheless active haptic devices could be made passive by standard control techniques and then the control scheme proposed in the paper can still be applied. Some extra energy injection can derive both from the Sample \& Hold device ( $S H$ in Fig. 8) and from the discrete virtual environment.

Loosely speaking, both Hannaford's and Colgate's approach consists in adding a layer between the virtual environment and the haptic device. This extra layer has to dissipate the extra energy that can be introduced either from the hold device or from 
the discretization of a certain system to be used as a virtual environment.

In the scheme proposed in the paper each possible source of extra energy is implemented passively. The main advantage is that the evolution of the virtual environment, and not only its behavior at the power port, is controlled to be passive; the only unavoidable production of energy takes place when there is the energy leap. This minimizes the need of acting on the system through extra dissipating dynamic (i.e., energy book-keeping or extra damping on the haptic device), increasing thus the transparency of the haptic interface. Furthermore passivity is ensured independently of the sample period used to implement the virtual environment. The concepts illustrated in Section III can be used to implement an haptic display without the introduction of any extra layer to dissipate energy. By means of the port-Hamiltonian formalism and of the discretization algorithm reported in Section III-A it is possible to built a discrete passive model of any physical system. The Sample \& Hold algorithm reported in Section III-B can then be used to interconnect in an energetic consistent way (i.e., without the introduction of any extra energy) the continuous haptic display and the discrete virtual environment. The whole haptic chain will be sampled data passive independently of the sample period used to implement the discrete virtual environment (if good care is taken with energy book-keeping).

\section{A. Delayed Virtual Environments}

It can happen that the virtual environment dynamics are very complex and that the computation time needed to run its simulation exceeds the sample period. In these cases we speak of delayed virtual environment. Several researchers addressed this problem and various methodologies have been proposed: in [30] an extension of the virtual coupling technique, based on input and output strict passivity has been proposed, and in [31] a wave-model based approach has been proposed. The aim of this section is to analyze the effect of a delay on the output on the passivity of a port-Hamiltonian system both the interaction port is represented by a pair of power conjugated variables and in the case the interaction port is given by a pair of scattering waves.

Let us consider a port-Hamiltonian system represented by (2) where the power port by means of which the system energetically interacts with the rest of the world in represented by an effort/flow pair. Assume, furthermore, that the system has impedance causality (i.e., flow in/effort out). In case of delay we have that the output power variable at the interaction port is:

$$
e_{I \delta}(t)=e_{I}(t-\delta)
$$

The nondelayed system would be passive with respect to the input/output pair $\left(e_{I}, f_{I}\right)$. It can be easily proven that delay in the output destroys passivity of the port-Hamiltonian. In fact

$$
\begin{aligned}
P(t) & =-e_{I \delta}(t) f_{I}(t) \\
& =-(e_{I}(t)+\underbrace{e_{I \delta}(t)-e_{I}(t)}_{\bar{e}(t)})^{T} f(t) \\
& =\frac{d H}{d t}+\underbrace{f_{R}^{T}(t) R(x) f_{R}(t)-\bar{e}^{T}(t) f(t)}_{P_{\text {diss }}} .
\end{aligned}
$$

Specific choices of the input variable can lead to a negative value of $P_{\text {diss }}$ and therefore to a production of extra energy and to a loss of passivity.

We can therefore conclude that when there is a certain delay in the computation of the output (i.e., in case of delayed virtual environment), port-Hamiltonian systems with an interaction power port represented by an effort/flow pair cannot be safely used because they are not passive since the conjugated power variables are not time-collocated. Consider a power port $(e, f)$ where $e$ and $f$ represent an effort and a flow respectively. A power port represents an exchange of energy between the system and the rest of the world and (18) shows that this exchange can be equally represented both by an effort/flow pair and by scattering variables. A definition of passivity based on scattering variables can be given. If $(e, f)$ is the power port by means of which a system interacts with the rest of the world and if $\left(s^{+}, s^{-}\right)$is its scattering representation, we have that a system is passive iff $\exists \beta>0$ such that

$$
\int_{0}^{t} \frac{1}{2}\left\|s^{-}(\tau)\right\|^{2} d \tau \leq \int_{0}^{t} \frac{1}{2}\left\|s^{+}(\tau)\right\|^{2} d \tau+\beta .
$$

Loosely speaking, a system is passive iff the outcoming energy bounded by the incoming energy, namely iff there is no internal production of energy.

One could think to deal with delayed virtual environments by simply discretizing a port-Hamiltonian system with power variables as input/output and then to treat the delay in the same way as in telemanipulation. In Fig. 9 we can see how the problem can be tackled in telemanipulation. The power variables of the port of the port-Hamiltonian system are coded into scattering variables and then sent through the communication channel. The port-Hamiltonian system is passive and it has a passive behavior at the power port. By scatterizing the power port, and transmitting scattering waves, this passive behavior is simply conserved during the communication, independently of any delay.

Unfortunately, when dealing with delayed virtual environments, the power variables at the power port are not consistent and this causes a nonpassive behavior of the system at the port. If we used the scheme in Fig. 9, the scattering waves would simply replicate the nonpassive behavior of the systems, leaving the problem unsolved. The delay has to be treated therefore in a different way in case of delayed virtual environment. The reason of this discrepancy is that while in telemanipulation the problem concerning delay is in the transmission of power variables and, therefore, something external to the system, in case of delayed port-Hamiltonian systems the delay is intrinsic into the dynamics of the system, internal to the system, and the scattering framework has to be embedded in the model of the system in order to deal with this kind of delay in a similar way as in [14] for defining impedance matching.

In order to deal with this internal delay we will model a port Hamiltonian system modeling the power port $\left(e_{I}, f_{I}\right)$, by means of which it interacts with the rest of the world, by the corresponding equivalent scattering representation $\left(s_{I}^{+}, s_{I}^{-}\right)$. Since (18) holds, we can write

$$
P_{I}+P_{C}+P_{R}=\frac{1}{2}\left\|s_{I}^{+}\right\|^{2}-\frac{1}{2}\left\|s_{I}^{-}\right\|^{2}+e_{C}^{T} f_{C}+e_{R}^{T} f_{R}=0
$$




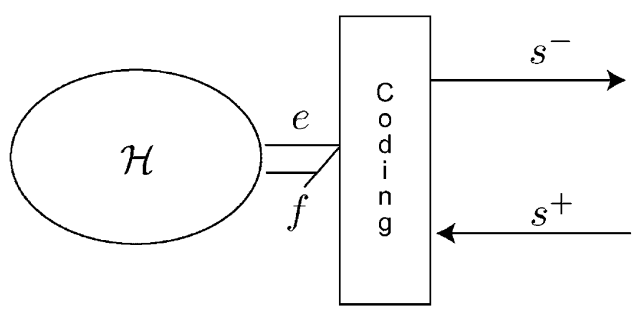

Fig. 9. Coding of power variables.

which is the power balance in terms of scatterized interaction port. By straightforward calculation we can get

$$
\left(\begin{array}{l}
s^{-} \\
f_{C}
\end{array}\right)=\left(\begin{array}{ll}
S_{1} & S_{2} \\
S_{3} & S_{4}
\end{array}\right)\left(\begin{array}{l}
s^{+} \\
e_{C}
\end{array}\right)
$$

where

$$
\begin{aligned}
& S_{1}=\left(B N^{-1}+N\right)^{-1}\left(B N^{-1}-N\right)^{-1} \\
& S_{2}=\sqrt{2}\left(B N^{-1}+N\right)^{-1} A \\
& S_{3}=\frac{C N^{-1}}{\sqrt{2}}\left(I-\left(B N^{-1}+N\right)^{-1}\left(B N^{-1}-N\right)\right) \\
& S_{4}=D-C N^{-1}\left(B N^{-1}+N\right)^{-1} A
\end{aligned}
$$

and now

$$
\dot{H}+f_{R}^{T} R(x) f_{R}=-\left(\frac{1}{2}\left\|s_{I}^{+}\right\|^{2}-\frac{1}{2}\left\|s_{I}^{-}\right\|^{2}\right)=P
$$

Let us now consider a port-Hamiltonian system in the form of (22) and suppose that there is a delay in the computation of the output. We have that

$$
\begin{cases}s_{I \delta}^{-}(t)=0 & t<\delta \\ s_{I \delta}^{\underline{-}}(t)=s_{I}^{-}(t-\delta) & t \geq \delta\end{cases}
$$

where we reasonably assumed that when the output is not yet ready because of the delay, the virtual environment presents 0 on the output buffer.

Proposition 3: The delayed scatterized port-Hamiltonian system is passive for any delay $\delta>0$.

Proof: Since the nondelayed system is passive we have that condition (20) holds and therefore that

$$
\int_{0}^{t} \frac{1}{2}\left\|s_{I}^{-}(\tau)\right\|^{2} d \tau \leq \int_{0}^{t} \frac{1}{2}\left\|s_{I}^{+}(\tau)\right\|^{2} d \tau+\beta \quad \forall t>0 .
$$

Let us now consider the delayed output $s_{I \delta}^{-}(t)=s_{I}^{-}(t-\delta)$. We have that

$$
s_{I \delta}^{-}(t)=0 \quad \forall t \in[0, \delta]
$$

We can write

$$
\begin{aligned}
\int_{0}^{t} \frac{1}{2}\left\|s_{I \delta}^{-}(\tau)\right\|^{2} d \tau & \leq \int_{0}^{t} \frac{1}{2}\left\|s_{I}^{-}(\tau)\right\|^{2} d \tau \\
& \leq \int_{0}^{t} \frac{1}{2}\left\|s_{I}^{+}(\tau)\right\|^{2} d \tau+\beta
\end{aligned}
$$

which implies

$$
\int_{0}^{t} \frac{1}{2}\left\|s_{I \delta}^{-}(\tau)\right\|^{2} d \tau \leq \int_{0}^{t} \frac{1}{2}\left\|s_{I}^{+}(\tau)\right\|^{2} d \tau+\beta
$$

and therefore the delayed system is passive.
Therefore, considering the scattering representation of the interaction power port, passivity is preserved even in case of delay on the output.

We can modify the passivity preserving discretization algorithm proposed in Section III-A in order to passively discretize a port-Hamiltonian system with scatterized interaction power port. If we rewrite (21) for the discrete case, we have

$\frac{1}{2}\left\|s_{I}^{+}(k)\right\|^{2}-\frac{1}{2}\left\|s_{I}^{-}(k)\right\|^{2}+e_{C}^{T}(k) f_{C}(k)+e_{R}^{T}(k) f_{R}(k)=0$.

We have that, during a sample period, the dissipated energy will be equal to $T f_{R}^{T}(k) R(x(k)) f_{R}(k)$ and the supplied energy will be equal to $-T\left((1 / 2)\left\|s_{I}^{+}(k)\right\|^{2}-(1 / 2)\left\|s_{I}^{-}(k)\right\|^{2}\right)$. In order to conserve passivity, similarly to what done in Section III.A we need a jump in internal energy $\Delta H(k)$ from instant $k T$ to instant $(k+1) T$ such that:

$$
\begin{aligned}
\Delta H(k)=-T f_{R}^{T}(k) R(x(k)) & f_{R}(k) \\
& -T\left(\frac{1}{2}\left\|s_{I}^{+}(k)\right\|^{2}-\frac{1}{2}\left\|s_{I}^{-}(k)\right\|^{2}\right)
\end{aligned}
$$

This implies that the new discrete state $x(k+1)$ should belong to an energetic level such that:

$$
H(x(k+1))=H(x(k))+\Delta H(k)
$$

The same considerations about the set of allowed states and about consistency with continuous dynamics made in Section III.A hold in this context. Hereafter we algorithmically explain the way the discrete system with scatterized power port can be integrated

1) Given an initial state $x(k)$, we set $e_{C}(k)=$ $(\partial H) /(\partial x)\left(x_{k}\right)$.

2) Using the value of the system input $s_{I}^{+}(k)$ and the previously calculated $e_{C}(k)$, we can calculate $s_{I}^{-}(k)$, the output of the interaction port, and $f_{C}(k)$ using the discrete representation of (22)

3) $f_{C}(k)$ is then used to calculate the next state $x(k+1)$ in the same way as in Section III-A.

In Fig. 10 is represented the general scheme for an intrinsically passive port-Hamiltonian based haptic interface.

Since Propostion 3 holds, the discrete system is passive (in a discrete sense) even in the output power wave is delayed because of computational delay. The interconnection between continuous and discrete domain is made through the element $S H$ which is the passive Sample \& Hold described in Section III-B. Since the interaction of the human operator with the virtual environment will have place through power variables (i.e., effort and flow) we endowed the scheme with a coding block which is used to interface the power variables based port with the scattering based port. In case (very frequent in haptics) that the virtual environment has an impedance causality, we have that from $f_{d}(k)$ and $s_{I}^{-}(k)$ we compute $s_{I}^{+}(k)$ and $e_{d}(k)$. Notice that now the coding procedure is safe. In fact the coding/decoding procedure is, by definition, such that the energetic behavior at the port 


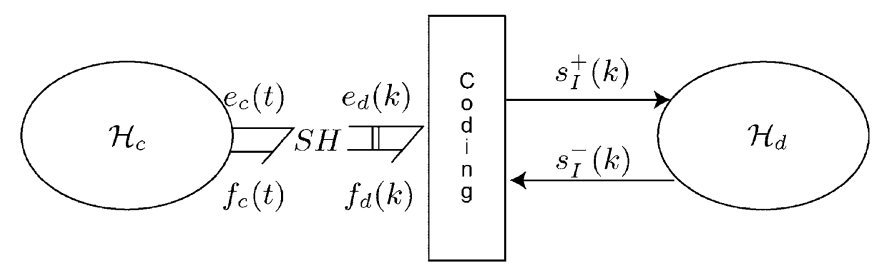

Fig. 10. Final scheme.

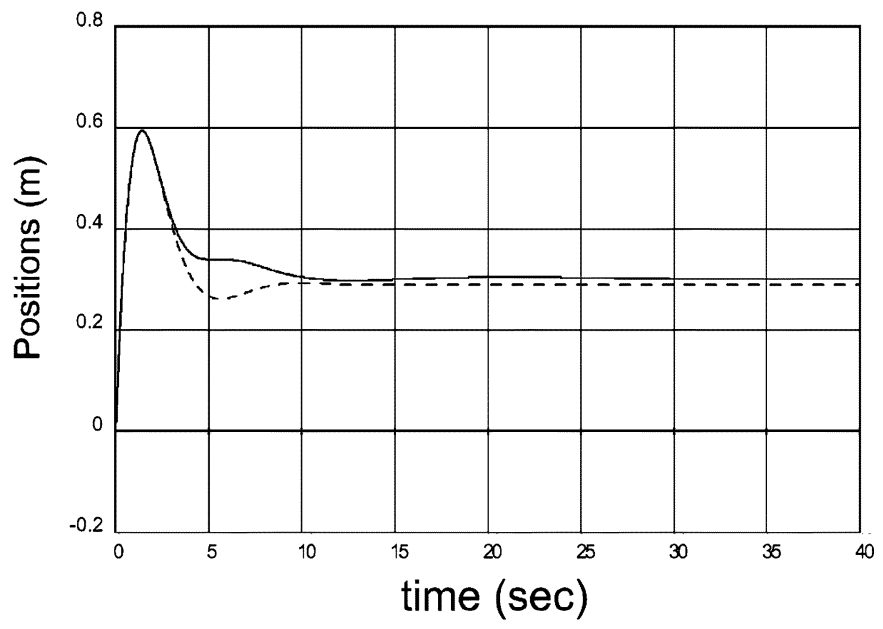

(a)

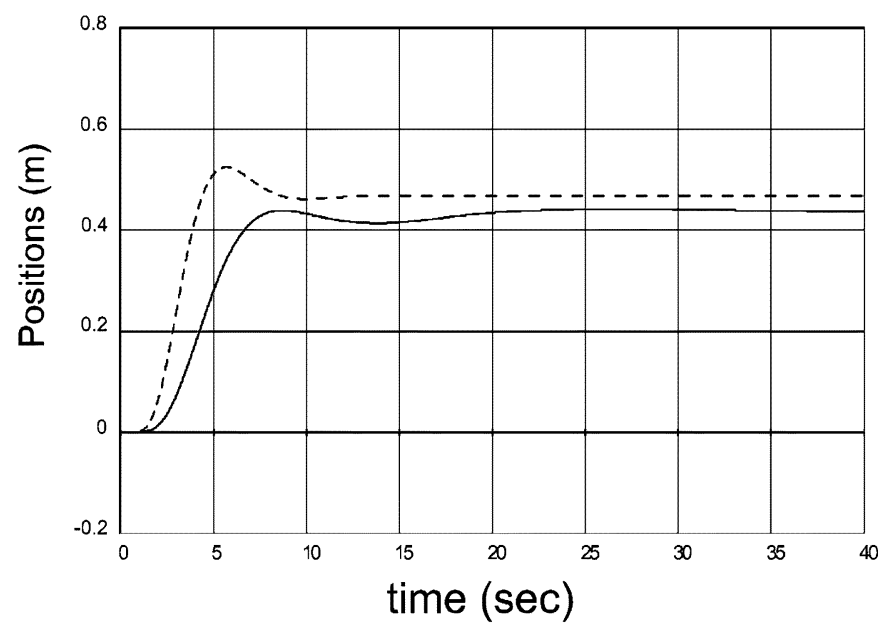

(b)

Fig. 11. Positions of master and slave in case an impulsive force is applied at the master side. The communication is characterized by variable delay and loss of packets. (a) Position of the Master. (b) Position of the Slave.

$\left(s_{I}^{+}, s_{I}^{-}\right)$and the one at the port $\left(e_{d}, f_{d}\right)$ are exactly the same (there is energetic synchronism); since Proposition 3 holds we have that the behavior at the port $\left(s_{I}^{+}, s_{I}^{-}\right)$is passive and therefore we will have a passive behavior at the port $\left(e_{d}, f_{d}\right)$ independently of any computational delay.

The proposed scheme provides a passive haptics scheme for delayed virtual environment without any extra layer to dissipate extra energy produced by the delay. The scheme is based on natural and intuitive energetic consideration and it has the advantage that transparency of the virtual environment is affected only by the unavoidable dynamical effect associated to the delay and it is not perturbed by any extra dynamics.

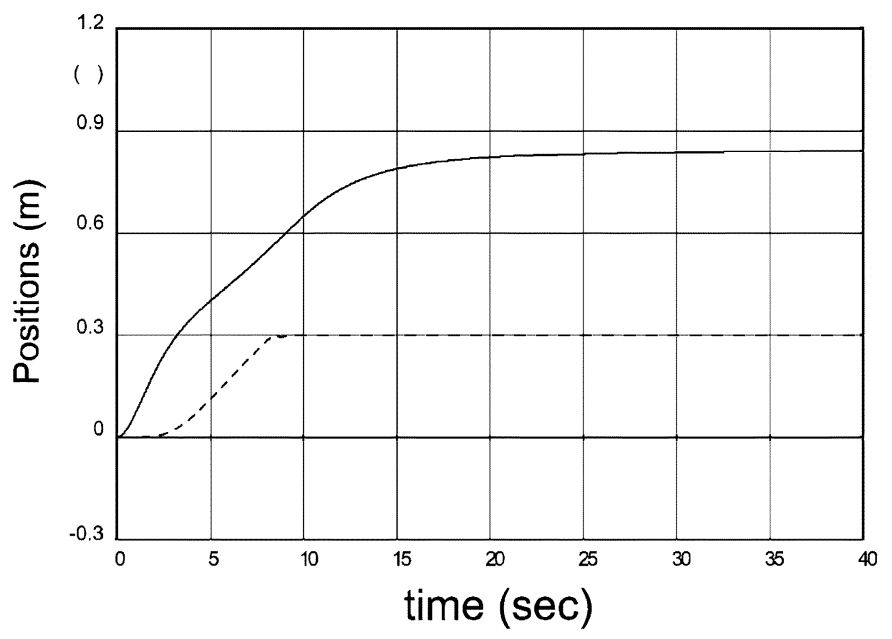

(a)

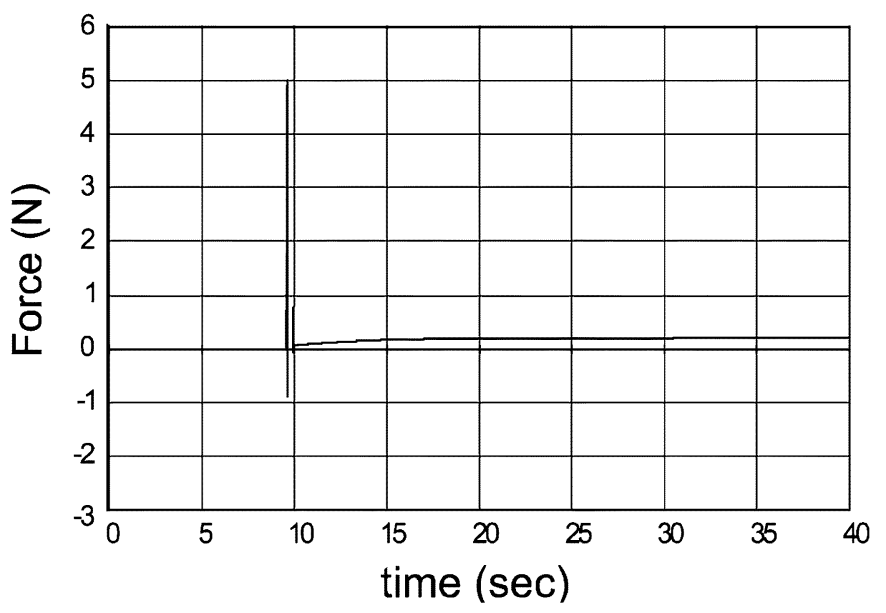

(b)

Fig. 12. Behavior of the system in case a constant force is applied on the maste and the slave interacts with a rigid wall. (a) Positions of the master (continuous) and of the slave (dashed). (b) Force applied on the master (solid) and force applied by the remote environment on the slave (dashed).

Remark 4: In case there is no computational delay on the output, we have that using either power variables or scattering waves leads to the same behavior of the system. The scheme in Fig. 10 can be, therefore, considered a generalization of the scheme proposed for nondelayed virtual environments.

Remark 5: Since the proposed haptics scheme is passive independently of the sample period, one could think to avoid the delayed output problem by simply increasing the sample period. This is true but performances of the system are affected by the sample period used: the higher is the sampling rate, the more realistic will be the simulations. In order to have a realistic haptic interface, therefore, it is necessary to keep the sample period as small as possible taking into account, in the implementation, possible computational delays.

\section{Simulations}

In this section, we will show some simulations in order to validate the results of the paper. Let us consider, first, a simple telemanipulation scheme built up using the concepts of the paper: each robot (master and slave) is a 1 DOF system, a simple mass, 


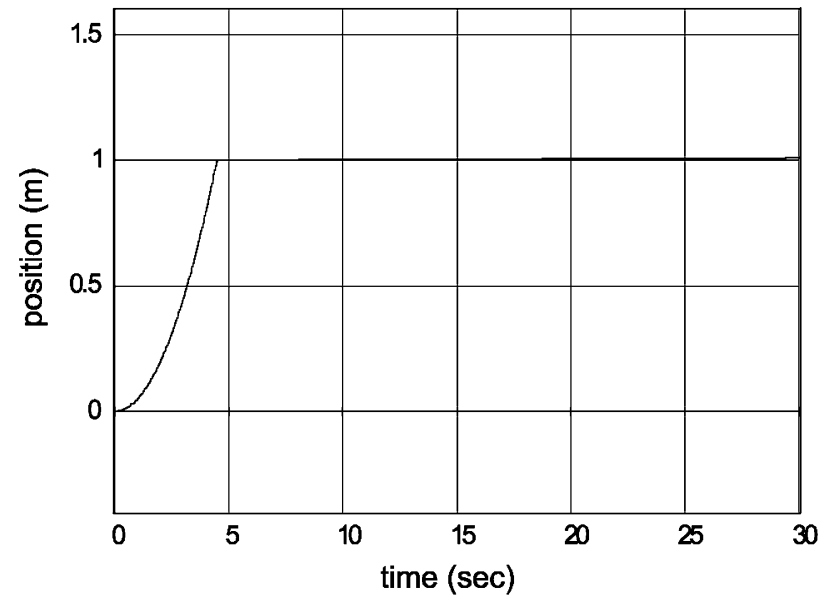

(a)

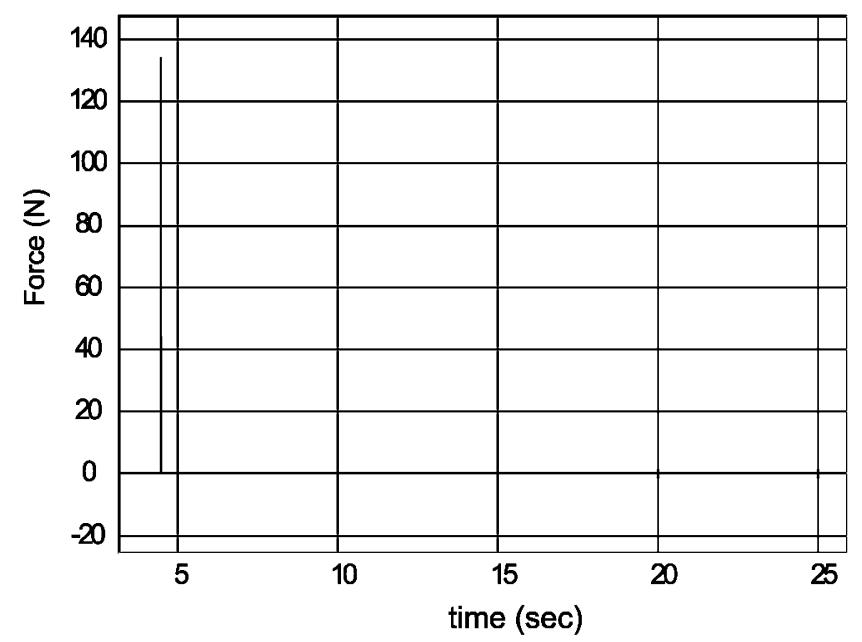

(b)

Fig. 13. Behavior of the system in case of interaction with a stiff virtual wall. (a) Position of the haptic device in case of interaction with a virtual wall set at $x=1 \mathrm{~m}$. (b) Force applied by the virtual wall on the haptic device.

and is controlled by a discretized IPC, connected in a power consistent way to the continuous robot; the sample period is $T=10$ msec. The communication channel is implemented by means of the digital scattering strategy. In the first simulation we implemented a variable transmission delay (with a mean of $0.5 \mathrm{sec}$.) and an unreliable communication: 10 packets are lost every $2 \mathrm{~s}$ and 20 packets every $3 \mathrm{~s}$ are lost in the communication between master and slave and slave and master respectively. In the first simulation the human operator applies an impulsive force at the master side; the behavior of the system is represented in Fig. 11. The dashed line represents the position of master and slave in case of constant delay $(0.5 \mathrm{~s})$ and of no loss of packets. We can notice that the performances decrease but the stability is maintained. In fact when packets are lost it means that some energy is not delivered either to the master or to the slave side. Performances in case of loss of packets are deteriorated since some of the energy required for performing the tracking task is lost in the communication. A possible way of improving performances is to interpolate the received packets in order to estimate the lost ones, as shown, for example, in [32].

In the next simulation we implemented an interaction task. The master is pushed with a constant force $F=0.1 \mathrm{~N}$ and the slave interacts with a wall (implemented with a spring-damper system, $K=1000 \mathrm{~N} / \mathrm{m}, b=100 \mathrm{Ns} / \mathrm{m}$ ) posed at position $x=0.3 \mathrm{~m}$; the communication channel used is the same of the previous simulation. The position of master and slave are shown in Fig. 12(a). We can see that the slave stops when it touches the wall. The force applied by the environment is reported in Fig. 12(b). The force applied by the remote environment exhibits a peak when the slave gets in touch with the remote environment and then the force applied by the environment becomes constant to equate the force applied on the master. The force of interaction is, therefore, reflected back to the master side and compensates the force applied to the master. In fact we can see that the position of the master is constant even if the operator is keeping on applying a force. The scheme exhibits therefore a good force reflection despite of the loss of packets in the communication.
The next kind of simulation is an haptic application of our scheme. We have a mass; a continuous haptic device, and a discrete virtual environment; a virtual wall. The amount of energy that can be introduced in the haptic interface because of the computational delay depends on the parameters characterizing the virtual environment. Very stiff and even very dissipative environments can inject big amounts of energy even for small computational delays. In order to prove the validity of the proposed scheme also in these cases, we implemented the virtual wall as a discrete port-Hamiltonian made up of a parallel of a very stiff spring $(k=100000 \mathrm{~N} / \mathrm{m})$ and of a large damper $(b=300$ $\mathrm{Nsec} / \mathrm{m})$. The simulation is one-dimensional: the wall is at the position $1 \mathrm{~m}$ and the haptic device is at an initial position $x_{0}=0$ $\mathrm{m}$ and is pushed by a constant force toward the wall; the sample frequency is $f=25 \mathrm{~Hz}$ and there is a computational delay of 3 sample periods. The virtual environment is obtained by means of passivity preserving discretization proposed in Section III.A while the computational delay is handled as explained in Section V.A. We can see from Fig. 13(a) that the position of the haptic device increases until it meets the wall; when the haptic device gets in touch with the virtual wall it stops when the force applied by the virtual wall balances the force applied to the system by the human operator. A stable behavior is achieved even if the sample time is quite big, the environment dynamics are quite fast and there is computational delay. The force exerted by the virtual wall is reported in Fig. 13(b). We can notice that when the haptic device gets in contact with the virtual wall there is a peak and after that the force tends to equate the action exerted by the operator on the haptic device.

\section{CONCLUSION}

In this paper, we have shown how it is possible to discretise a port-Hamiltonian system preserving, in a discrete sense, its passivity. Furthermore we described how to interconnect discrete and continuous port-Hamiltonian systems in an energetically consistent way. These concepts can be successfully applied both in telemanipulation and haptics. We have shown that 
it is possible to explicitly take into account the discrete nature of IPCs and packet switching communication channels in geometric telemanipulation. Passivity of the telemanipulation is granted independently of the sample period and of variable delay and loss of packets in the transmission line. Furthermore we can use the proposed techniques to obtain an haptic display which is passive for any physical virtual environment and for any sample period. Moreover, by using scattering formalism, we can handle also computational delays in virtual environments. Future work will be focused on practical experimentation of these techniques both to telemanipulation techniques and to haptic interfaces.

\section{REFERENCES}

[1] S. Stramigioli, C. Secchi, A. van der Schaft, and C. Fantuzzi, "A novel theory for sample data systems passivity," in Proc. IEEE Conf. Intelligent Robotic Systems, 2002.

[2] S. Stramigioli, "About the use of port concepts for passive geometric telemanipulation with varying time delays," in Proc. Mechatronics Forum Int. Conf., Enschede, The Netherlands, Jun. 2002.

[3] C. Secchi, S. Stramigioli, and C. Fantuzzi, "Digital passive geometric telemanipulation," in Proc. IEEE Int. Conf. Robotics Autom., Taipei, Taiwan, Sep. 2003.

[4] B. Hannaford et al., "Time domain passivity control of haptic interfaces," IEEE Trans. Robot. Automat., vol. 18, no. 1, pp. 1-10, Feb., 2002.

[5] R. Anderson and M. Spong, "Bilateral control of teleoperators with time delay," IEEE Trans. Automat. Contr., vol. 34, no. 5, pp. 494-501, May 1989.

[6] G. Niemeyer and J. Slotine, "Stable adaptive teleoperation," IEEE J. Oceanic Eng., vol. 16, no. 1, pp. 152-162, Jan. 1991.

[7] T. Courant, "Dirac manifolds," Trans. Amer. Math. Soc. 319, pp 631-661, 1990.

[8] A. van der Schaft and B. Maschke, "The Hamiltonian formulation of energy conserving physical systems with external ports," Arch. Elekt. Übertragung. , vol. 49, no. 5/6, pp. 362-371, 1995.

[9] A. van der Schaft, M. Dalsmo, and B. Maschke, "Mathematical structure in the network represenation of energy-conserving physical systems," in Proc. IEEE Conf. Decision Control, Kobe, Honshu, Japan, Dec. 1996.

[10] A. Bloch and P. Crouch et al., "Representation of dirac structures on vector spaces and nonlinear lcv-circuits," in Proc. Pure Mathematics Differential Geometry Control Theory Symp. , vol. 64, H. Ferraya et al., Eds., 1999, pp. 103-117.

[11] S. Stramigioli, Modeling and IPC Control of Interactive Mechanical Systems: A Coordinate Free Approach, ser. LNCIS. Berlin, Germany: Springer-Verlag, 2001

[12] G. Golo, S. Stramigioli, and A. van der Schaft, "Hamiltonian formulation of planar beams," in Proc. 2nd Workshop Lagrangian Hamiltonian Methods Nonlinear Control. Sevilla, Spain, Apr. 2003, pp. 169-174.

[13] H. Paynter, Analysis and Design of Engineering Systems. Cambridge, MA: MIT Press, 1960.

[14] S. Stramigioli, A. J. van der Schaft, B. Maschke, and C. Melchiorri, "Geometric scattering in robotic telemanipulation," IEEE Trans. Robot. Automat., vol. 18, no. 4, Apr. 2002.

[15] R. Horowitz and P. Li, "Passive velocity field control (PVFC) Part I Geometry and robustntess," IEEE Trans. Automat. Contr., vol. 46, no. 9, Sep. 2001.

[16] S. Stramigioli, "From differentiable manifolds to interactive robot control," Ph.D. dissertation, Delft Univ. Technology, Delft, The Netherlands, 1998.

[17] A. van der Schaft, $L_{2}$-Gain and Passivity Techniques in Nonlinear Control. Berlin, Germany: Springer-Verlag, 2000.

[18] B. Maschke and A. van der Schaft, "Port controlled hamiltonian representation of distributed parameter sytems," in Proc. Workshop Modeling Control Lagrangian Hamiltonian Systems, 2000.

[19] Y. Yokokohji, T. Imaida, and T. Yoshikawa, "Bilateral control with energy balance monitoring under time-varying communication delay," in Proc. IEEE Int. Conf. Robotics Autom., San Francisco, CA, Apr. 2000.

[20] Y. Yokokohji, T. Tsujioka, and T. Yoshikawa, "Bilateral control with time-varying delay including communication blackout," in Proc. 10th Symp. Haptic Interfaces Virtual Environments Teleoperator Systems, 2002.

[21] N. Chopra, M. Spong, S. Hirche, and M. Buss, "Bilateral teleoperation over the internet: The time varying delay problem," in Proc. American Control Conf., vol. 1, Denver, CO, Jun. 2003.
[22] S. Hirche and M. Buss, "Passive position controlled telepresence systems with time delay," in Proc. American Control Conf., vol. 1, Denver, CO, Jun. 2003.

[23] C. Secchi, S. Stramigioli, and C. Melchiorri, "Geometric grasping and telemanipulation," in Proc. IEEE Conf. Intelligent Robotic Syst., Maui, HI, Oct. 2001.

[24] C. Melchiorri and A. Eusebi, Modeling and Control of Mechanisms and Robots: World Scientific, 1996, ch. Telemanipulation: System Aspect and Control Issues.

[25] P. Arcara and C. Melchiorri, "A comparison of control schemes for teleoperation with time delay," in Proc. Conf. Telematics Applications, 2001, pp. 86-92.

[26] N. Hogan, "Controlling impedance at the man/machine," in Proc. IEEE Int. Conf. Robotics Autom., 1989, pp. 1626-1631.

[27] J. Colgate and G. Schenkel, "Passivity of a class of sampled-data systems: Application to haptic interfaces," in Proc. American Control Conf., 1994.

[28] B. Miller, J. Colgate, and R. Freeman, "Guaranteed stability of haptic systems with nonlinear virtual environnments," IEEE Trans. Robot. Automat., vol. 16, no. 6, Dec., 2000.

[29] S. Salcudean and T. Vlaar, "On the emulation of stiff walls and static friction with a magnetically levitated input-output device," ASME J. Dynamic Systems, Measurement Control, vol. 119, Mar. 1997.

[30] B. Miller, J. Colgate, and R. Freeman, "Environment delay in haptic systems," in Proc. IEEE Int. Conf. Robotics Autom., San Francisco, CA, Apr. 2000.

[31] H. Arioui, A. Kheddar, and S. Mammar, "A predictive wave-based approach for time delayed virtual environments haptics systems," in Proc. 2002 IEEE Int. Workshop Robot Human Interactive Communication, Berlin, Germany, Sep. 2002.

[32] C. Secchi, S. Stramigioli, and C. Fantuzzi, "Dealing with unreliabilities in digital passive geometric telemanipulation," in Proc. IEEE/RSJ Int.Conf. Intelligent Robots Systems, Las Vegas, NV, Oct. 2003.

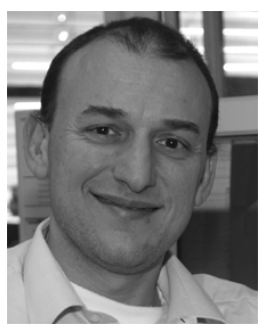

Stefano Stramigioli (SM'00) received the M.Sc. degree with honors (cum laude) rom the University of Bologna, Bologna, Italy, in 1992 and the Ph.D. degree with honors (cum laude) from the Delft University of Technology, Delft, in 1998.

Since 1998 he has been first an Assistant Professor with the Delft University of Technology, Delft, the Netherlands and then Associate Professor with the University of Twente, Twente, The Netherlands. He has more than 50 publications including a book. He is involved in different projects related to control, robotics, MEMS, and intelligent transportation systems and Coordinator of the European Project Geoplex (http://www.geoplex.cc). He has been teaching modeling, control, and robotics for under- and post-graduates and has received teaching nominations and an award.

Dr. Stramigioli is currently the Vice President for Technical activities of the Intelligent Transportation Systems Society, and the IEEE Representative of IEEE RAS for ITSC. He is chairing the Technical Committee on Intelligent Transportation Systems for the IEEE Robotics and Autom. Society. He is a member of the ESA Topical Team on Dynamics of Prehension in Micro-gravity and its application to Robotics and Prosthetics. He is Editor-in-Chief of the IEEE ITSC Newsletter, and the IEEE Robotics and Autom. Magazine and has been Guest Editor for others.

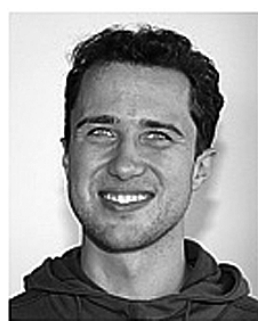

Cristian Secchi (M'00) received the M.Sc. degree in computer science engineering from the University of Bologna, Bologna, Italy, in 2000. He received the $\mathrm{Ph} . \mathrm{D}$. degree from the University of Modena and Reggio Emilia, Italy, in 2004.

In 2000, he was with the Delft University of Technology, Delft, The Netherlands, for a three-month internship under the supervision of Prof. S. Stramigioli. In 2001, he was with the Department of Sciences and Methods of the Engineering at the University of Modena and Reggio Emilia, Italy, as a Ph.D. student under the supervision of Prof. C. Fantuzzi. In 2002 he was with the University of Twente, The Netherlands, as a Visiting Scholar at the Control group, working with Prof. S. Stramigioli and Prof. A. van der Schaft. Since 2004, he has been a Researcher at the Department of Science and Methods of the Engineering at the University of Modena and Reggio Emilia. His reasearch interests are passivity theory and passive control, port-Hamiltonian systems, haptics, and telemanipulation. 


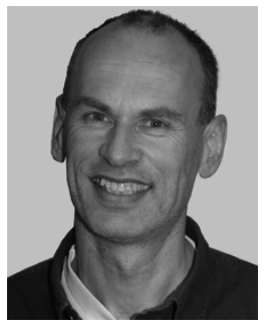

Arjan J. van der Schaft (F'00) received the undergraduate and $\mathrm{Ph} . \mathrm{D}$. degrees in mathematics from the University of Groningen, The Netherlands, in 1979 and 1983, respectively.

In 1982 he joined the Department of Applied Mathematics, University of Twente, Enschede, The Netherlands, where he is presently a full professor in Mathematical Systems and Control Theory. His research interests include the mathematical modeling of physical and engineering systems and the control of nonlinear and hybrid systems. He has served as Associate Editor for Systems \& Control Letters, Journal of Nonlinear Science, and SIAM Journal on Control. Currently he is Associate Editor for Systems and Control Letters and Editor-at-Large for the European Journal of Control. He is author or coauthor of the following books: System Theoretic Descriptions of Physical Systems (Amsterdam, The Netherlands: CWI, 1984), Variational and Hamiltonian Control Systems with P. E. Crouch (Berlin, Germany: Springer-Verlag, 1987), Nonlinear Dynamical Control Systems with H. Nijmeijer (Berlin, Germany: Springer-Verlag, 1990), $L_{2}$-Gain and Passivity Techniques in Nonlinear Control (Berlin, Germany: Springer-Verlag, 2000), and An Introduction to Hybrid Dynamical Systems with J.M. Schumacher (Berlin, Germany: Springer-Verlag, 2000).

Dr. van der Schaft is the Editor of the IEEE TRANSACTIONS ON AUTOMATIC CONTROL.

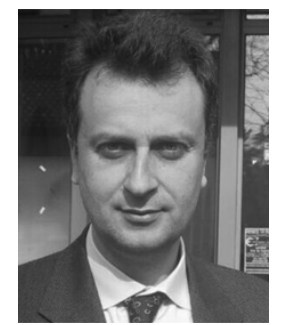

Cesare Fantuzzi (M'00) received the Laurea degree in electrical engineering and the Ph.D. degree in system engineeering on the topic of "fuzzy control," all from the Universita' di Bologna, Bologna, Italy, in 1990 and 1995, respectively.

In 1996, he became Assistant Professor at the 'Dipartimento di Ingegneria', Universita' di Ferrara and, since 2001, he is an Associate Professor at the 'Dipartimento di Scienze e Metodi per l'Ingegneria', Universita' di Modena e Reggio Emilia. He teaches courses in control system and industrial automation. He has published papers on fuzzy control, advanced mechatronics, fault diagnosys, and telemanipulation. 